\title{
Morphological Features of the Pili Associated with Escherichia coli K 12 Garrying R Factors or the F Factor
}

\author{
By A. M. LAWN \\ Department of Electron Microscopy, Lister Institute of \\ Preventive Medicine, Chelsea Bridge Road, London, S.W.1
}

(Received 21 April 1966)

\begin{abstract}
SUMMARY
Strains of Escherichia coli $\mathrm{x} 12$ carrying various R factors or the $\mathrm{F}$ factor were examined with the electron microscope. The $\mathbf{F}$ specific RNA phage MS2 was added to differentiate the specific $F$ type pili, which adsorbed the phage, from common pili, which did not. Even when phage was not added it was possible to identify $F$ type pili because they were wider and generally longer than common pili. F type pili, but never common pili, often possessed terminal knobs whose presence on attached pili has not been previously described. The F type pili formed by $\boldsymbol{E}$. coli carrying the different $\mathbf{R}$ factors or $\mathrm{Hfr} H$ could not be morphologically distinguished from each other. Only one morphological type of common pilus was observed.
\end{abstract}

\section{INTRODUCTION}

Crawford \& Gesteland (1964) first reported the presence of pili on male $\left(\mathrm{F}^{+}\right)$but not on female $\left(\mathrm{F}^{-}\right)$bacteria which could adsorb the male-specific RNA bacteriophage R 17. Brinton, Gemski \& Carnahan (1964) showed that the phage receptive pili were of a special type, wider and longer than common pili (fimbriae: Duguid \& Wilkinson, 1961; type I pili: Brinton, 1965) and named them F pili. Subsequently it was demonstrated that susceptibility to male-specific phages and the ability to conjugate and act as a genetic donor were both related to the presence of $F$ pili (Valentine, Wedel \& Ippen, 1965; Brinton, 1965).

Certain drug resistance factors ( $\mathbf{R}$ factors) responsible for transmissible resistance to antibacterial agents also determine the production of pili with affinity for malespecific RNA phage (Datta, Lawn \& Meynell, 1966). In a given $\mathbf{R}^{+}$culture, the proportion of bacteria with these $\mathbf{F}$ type pili was directly related to the proportion which could be infected with the phage, and to the proportion able to transmit their factor to suitable recipients.

In the present paper pili with affinity for $\mathbf{F}$ specific phage are referred to as ' $\mathbf{F}$ type pili' whether present on bacteria carrying the $\mathbf{F}$ factor itself or an $\mathbf{R}$ factor. Common pili are those which do not absorb the phage. A detailed examination has been made of the pili present on $\mathrm{F}^{+}$and $\mathrm{R}^{+}$bacteria in order to compare $\mathbf{F}$ type pili determined by $\mathbf{F}$ itself with those determined by various $\mathbf{R}$ factors, and to contrast these with the common pili present in many enterobacteria (Duguid \& Wilkinson, 1961). 


\section{METHODS}

The strains of bacteria, $\mathbf{R}$ factors and preparation of the samples are described in Datta et al. (1966). The bacteria were all derivatives of Escherichia coli $\mathrm{K} 12$ carrying either the $\mathbf{F}$ factor ( $\mathbf{H f r} H$ ) or one of the three $R$ factors, $R 1, R 124$, or $\mathbf{R 2 3 7}$. Phage-free suspension and suspensions that had been mixed with the F-specific RNA phage MS 2 at a multiplicity of about 500 plaque-forming particles/bacterium were examined.

Electron microscopy. A modification of the method of Brenner \& Horne (1959) was used. A drop of the formalin-fixed culture was placed on a formvar-coated carbon-supported copper grid and either washed after it had remained for 2-3 min., or dried and then washed. The contrast agent was applied while the grid was still wet after washing. After a few seconds most of the remaining fluid was removed and the grid allowed to dry. Uranyl acetate was found to be the most satisfactory contrast agent.

The preparations were examined in a Philips EM200 microscope with $80 \mathrm{kV}$. accelerating voltage and a $25 \mu$ objective aperture with the double condenser. The magnification of the instrument was calibrated by means of a diffraction grating replica: the calibration was reproducible to within $5 \%$.

\section{RESULTS}

\section{Identification of common pili}

No pili absorbing the bacteriophage MS2 could be seen in $\mathbf{F}-\mathbf{R}$ - cultures of Escherichia coli к 12. Piliated bacteria were usually present, but all the pili were of one type, whose lengths and diameters were characteristic of common (type I) pili (see Brinton, 1965).

\section{Identification of $F$ type pili}

In strains of Escherichia coli $\mathrm{k} 12$ carrying R1, R124 or R237 and in strain $\mathrm{Hfr} \mathbf{H}$ the phage MS2 clearly distinguished common pili from $\mathrm{F}$ type pili, which were coated with the small spherical phage particles (Pl. 1, fig. 2). Even in cultures without MS 2, F type pili were readily distinguishable, being wider and generally longer than common pili (Pl. 1, fig. 1). F type pili, but not common pili, often possessed terminal knobs. Although adsorption of $\mathrm{F}$ specific phage must remain the final criterion for the identification of $\mathbf{F}$ type pili, these morphological features are further evidence for the structural dissimilarity between $\mathbf{F}$ type pili and common pili.

(a) Diameters of $F$ type and common pili. Measurements of $F$ type pili could be made only on bacteria from cultures without phage MS2 otherwise the phage particles obscured the true diameter of the pilus. In any one area of a micrograph of a culture without phage, $\mathrm{F}$ type pili are clearly wider than common pili (Pl. 1, fig. 1; Pl. 2, fig. 3b; Pl. 4, fig. 10). However, the negative contrast technique, with its irregular distribution of stain, does not give uniform effects from one micrograph to another, nor even over different areas of a single micrograph. Measurements of pilus diameters over several micrographs therefore vary over a considerable range; common pili in some areas may be as broad as $\mathbf{F}$ type pili in others. The point is illustrated in Fig. 1 where the maximum and minimum diameters of individual 
F type pili and neighbouring common pili are shown diagrammatically. The extreme range of diameter throughout the clearly outlined portion of an individual $F$ type pilus (recognized either by a terminal knob or by contrast with the diameter of neighbouring common pili) on a single micrograph was measured. One or more common pili in the vicinity were similarly measured.

Although the diameters of common pili occasionally lay within the range of an F type pilus, the values for the two kinds of pili fall clearly into two groups. All pili with terminal knobs were within the $\mathbf{F}$ type diameter-range. There is no evidence for the existence of more than one kind of $F$ type pilus or of one kind of common (type 1) pilus.

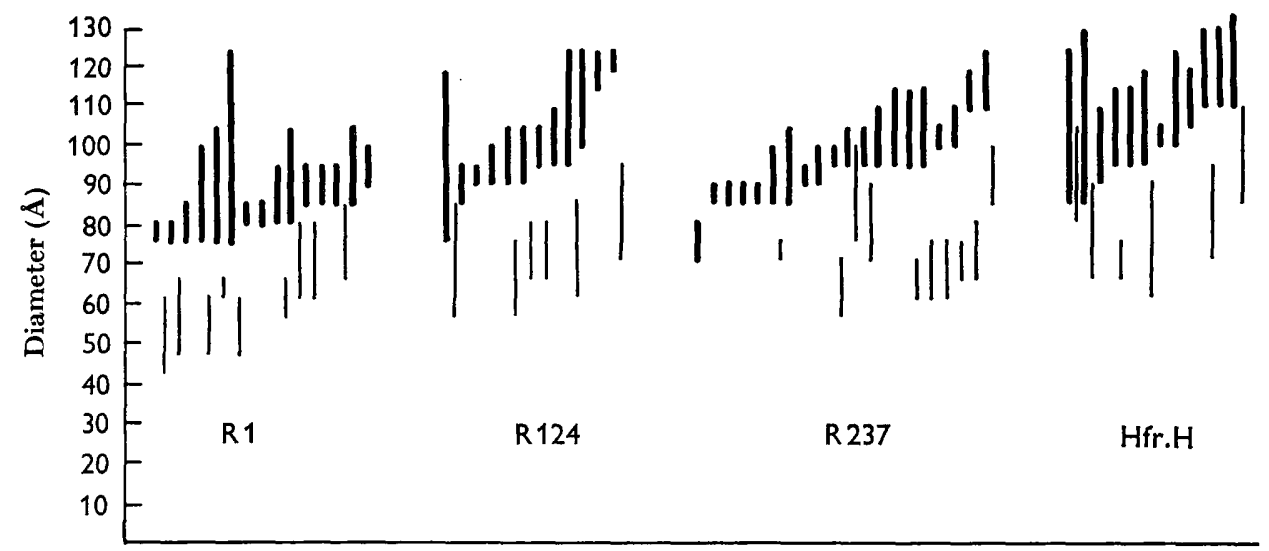

Fig. 1. The range of diameters of $F$ type pili and common (type I) pili formed by Escherichia coli $\mathrm{k} 12$ carrying different conjugation factors. A thick vertical line represents the diameter range of an individual $F$ type pilus. The thin line immediately to its right represents the extreme range of diameters of one or more common pili in the vicinity of that $F$ type pilus. The measurements are grouped according to the conjugation factor concerned.

Although (Fig. 1) the range of diameters of common (type 1) pili is large (45$100 \AA$ ), the distribution is in agreement with the figure of $70 \AA$ deduced by Brinton (1965) from X-ray diffraction analysis and from measurements of shadowed preparations in the electron microscope. The average diameter (95 $\AA$ ), and range (75-135 $\AA$ ) of $\mathrm{F}$ type pili do not agree so well with the diameter of $85 \AA$ given by Brinton (1965), who did not describe the method by which he arrived at this figure. Indeed, in the present sample, only $25 \%$ of $\mathrm{F}$ type pili had minimum diameters of $85 \AA$ or less.

Both $\mathbf{F}$ type and common pili had a central dense line when prepared by the negative contrast technique. In micrographs taken near to true focus this dense line had a maximum diameter of approximately $15 \AA$ in both types of pili. It was less prominent in $\mathbf{F}$ type pili, probably because of their greater thickness.

(b) Terminal knobs of $F$ type pili. In a preparation containing phage, many of the pili which adsorbed $F$ specific phage were enlarged at their distal extremities. Knobs were present on both short and long $\mathbf{F}$ type pili, but never on common pili. They did not result from the attachment of the phage, for the same knobs were seen in preparations to which no phage had been added. The shape and size of terminal 
knobs was variable but they can be divided into three main types: disc-shaped, cup- or flask-shaped and spherical, The spherical knobs were vesicular, vesicular with a solid centre (sometimes laminated) or completely solid in appearance (Pl. 2, figs. $3 a-f$ and fig. 4). The disc-shaped vesicular and solid knobs were respectively about 150-200, 400-800 and 250-700 $\AA$ in diameter. The spherical knobs are the most common. In some instances the $F$ type pilus appeared to taper as it approached the knob, particularly in the case of the small solid knobs; this may be a staining artefact.

(c) Lengths of $F$ type and common pili. $F$ type pili associated with each of the conjugation factors varied in length from a fraction of a micron to over $20 \mu$; common pili seldom exceeded $\mathbf{1 . 5} \mu$ in length.

\section{Other features of common pili}

In the strains studied, common piliation is not exclusively an all-or-none condition, as suggested in a recent review by Brinton (1965). Although the number of common pili per bacterium is most frequently zero or very large, many bacteria have only a few common pili. However, the proportion of bacteria bearing these pili varies considerably in different preparations of the same culture or even in different areas of the same grid.

Frequently, fragments of common pili broken during the preparation for electron microscopy were joined by a fine thread 15-25 $\AA$ in diameter (Pl. 4, fig. 8).

\section{Association of common with $F$ type pili}

Some bacteria bearing $\mathbf{F}$ type pili without the phage MS2 label had a curious elongated structure extending from the bacterium or joining one bacterium to another (PI. 3, fig. 5). At higher magnification this was resolved into a bundle of parallel filaments with the diameter of common pili. However, the length of these bundles exceeded that of a single common pilus. These bundles are formed by the deposition of detached common pili on the surface of an $F$ type pilus, which is sometimes seen protruding from the end (Pl. 3, fig. 6). As the common pili are so short, they must lie in series to form a continuous sheath. A similar association of the two types of pili occurred in phage-labelled cultures, but less frequently and to a lesser degree. Where a common I pilus ran along the side of an $F$ type pilus no phage particles were adsorbed on that side (Pl. 4, fig. 9).

In preparations of both labelled and unlabelled cultures bundles consisting solely of common I pili often occurred. Where there was more than one layer, pili in the second row often lay in the grooves between adjacent pili of the first row. This alignment gave rise to a heightening of contrast (Pl. 4, fig. 7) which appears to result from a re-inforcement of the dense central lines of the first row by superimposition of stain deposits at the edge of the second row.

\section{Association of phage particles with $F$ type pili}

The number of phage particles attached to a $2000 \AA$ length of $F$ type pilus was measured in cultures of organisms carrying different conjugation factors. In most cases the average number was close to 15 (range $11-20,75$ particles $/ \mu$ of pilus). With the ratio of phage particles to bacteria employed (about $500: 1$ ) this figure 
was relatively constant, in spite of wide differences in the proportion of $\mathbf{F}$ type piliated bacteria. A calculation, based on the minimum distance between phage particles, suggested that 125-150 particles could lie in contact with each micron of pilus if they were closely packed and if the whole surface of the pilus was receptive. The observed average of 75 particles $/ \mu$ although considerably lower than this figure, more probably results from restriction of ordered packing by random arrival of particles than of restriction of receptor sites on the pilus. In cultures to which a small number of phage particles were added it was clear that phage adsorption was initially random. With large numbers of phage particles the disposition along the pilus was not ordered.

\section{DISCUSSION}

In Escherichia coli $\mathrm{K} 12$, the $\mathbf{F}$ type pili associated with the $\mathbf{R}$ factors $\mathbf{R} 1, \mathbf{R} 124$ and $\mathbf{R 2 3 7}$ could not be distinguished morphologically from those produced by Hfr H, which carries the $\mathbf{F}$ factor itself. There was no consistent difference either in diameter or length, and some of each kind had terminal knobs. The constancy of the average number of phage MS 2 particles adsorbed per unit length of $\mathbf{F}$ type pilus formed by different strains is another point of similarity between them. All could readily be distinguished from common (type 1) pili.

Anderson \& Strand (1965) found spherical bodies on preparations of detached F pili, and considered these to be basal structures or roots. Although basal structures can be seen on non-attached flagella, they cannot be seen on the free ends of flagella which are still attached to the bacterium (Abram, Koffler \& Vatter, 1965). On the contrary, the terminal knobs of $F$ type pili are as frequently present on the free end of attached pili as on non-attached pili. On some bacteria as many as 4 out of $5 \mathrm{~F}$ type pili are terminated by knobs (Pl. 2, fig. 4). For this reason, the knobs are unlikely to be basal root-like structures. There are two other possibilities. First, these structures may be present on the $\mathbf{F}$ type pili when they first emerge from the bacterial cell wall, possibly representing an extrusion of part of the cell wall/plasma membrane complex with the emerging tip. Secondly, the distal extremity of the $\mathbf{F}$ type pilus may have a special affinity for cell-wall material, associated with its supposed function in conjugation, and that stable association occurs with particles of cell wall complex present in the culture. Structures resembling terminal knobs occur from time to time in lysates of enterobacteria infected with various phages as well as in phenol-extracted cell wall preparations from enterobacteria (Lawn \& Wilkinson, unpublished observations).

The bizarre structures resulting from association of common pili and F-type pili are described because they might cause difficulties in interpretation. The association is probably merely an expression of the general tendency of common pili to adhere to other structures (Duguid \& Gillies, 1957, 1958) and does not necessarily imply any surface structural relationships as in the case of the single-layered and multiplelayered structures found in purified preparations of pili (Brinton, 1965). The lesser degree of association in phage-labelled cultures suggest that it is a slow process, most of it probably occurring after the addition of phage to the culture. An alternative but unlikely explanation is that the phage particles can displace the associated common pili from $\mathbf{F}$ type pili. 
The author would like to thank the Fleming Memorial Fund for Medical Research which provided the electron microscope and additional support.

\section{REFERENCES}

Abram, D., Kofflep, H. \& Vatter, A. E. (1965). Basal structure and attachment of flagella in cells of Proteus vulgaris. J. Bact. 90, $137 \%$.

Anderson, T. F. \& Strand, M. (1965). Complexes of F pili and RNA bacteriophages. Science 148, 511.

Brenner, S. \& Horne, R. W. (1959). A negative staining method for high resolution electron microscopy of viruses. Biochim. biophys. Acta 34, 103.

Brinton C. C. Jun. (1965). The structure, function, synthesis and genetic control of bacterial pili and a molecular model for DNA and RNA transport in gram negative bacteria. Trans. N.Y. Acad. Sci. 27, 1003.

Brinton,C. C. Jun., Gemski, P. Jun. \& Carnahan, J. (1964). A new type of bacterial pilus genetically controlled by the fertility factor of $E$. coli $\mathrm{K} 12$ and its role in chromosomal transfer. Proc. natn. Acad. Sci. U.S.A. 52, 776.

Crawford, E. M. \& Gesteland, R. F. (1964). The absorption of bacteriophage R-17. Virology 22, 165.

Datta, N., LAwn, A. M. \& Meynelx, E. (1966). The relationship of F-type piliation and F phage sensitivity to drug resistance transfer in $\mathbf{R}^{+} \mathbf{F}^{-}$Escherichia coli $\mathrm{k} 12$. J. gen. Microbiol. 45, 365.

Duguid, J. P. \& Gillies, R. R. (1957). Fimbriae and adhesive properties in dysentery bacilli. J. Path. Bact. 74, 397.

Duguid, J. P. \& Gilites, R. R. (1958). Fimbriae and haemagglutinating activity in Salmonella, Klebsiella, Proteus and Chromobacterium. J. Path. Bact. 75, 519.

Duguid, J. P. \& Wilkinson, J. F. (1961). Environmentally induced changes in bacterial morphology. Symp. Soc. gen. Microbiol. 11, 69.

VAlentine, R. C., Wedel, H. \& IPPEN, K. A. (1965). F-pili requirements for RNA bacteriophage adsorption, Biochim. biophys. Acta 21, 277.

\section{EXPLANATION OF PLATES}

All the micrographs are of Escherichia coli $\mathrm{k} 12$ carrying one of the $R$ factors $R 1, \mathbf{R} 124$ or $R 237$. In some cases the $\mathrm{F}$ specific RNA phage MS2 was added to identify the $\mathrm{F}$ type pili. Preparations were made by the negative staining technique with uranyl acetate except fig. 10 for which uranyl formate was used.

\section{Plate 1}

Fig. 1. E. coli $\times 12, \mathrm{R} 124$ without RNA phage MS2. A single flagellum and many pili are present. In this instance the $\mathrm{F}$ type pilus is hardly longer than neighbouring common pili, yet it can be distinguished because of its greater diameter, shown in (b) which is an enlargement of the area outlined in $(a)$.

Fig. 2. $E$. coli $\times 12$ R287 mixed with the RNA phage MS2. The F type pilus, which has absorbed the round phage particles, can easily be distinguished from the common pili, which have no absorbed phage. Two flagella can be recognized by their width and distinctive surface structure.

\section{Plate 2.}

Fig. 3. Terminal knobs of $F$ type pili from $E$. coli $\mathrm{k} 12$ carrying various $\mathrm{R}$ factors, some with $\mathrm{F}$ specific phage particles. The calibration bar in (c) applies to all parts of the figure.

Fig. 4. A group of $5 \mathrm{~F}$ type pili attached to $E$. coli $\mathrm{K} 12 \mathrm{R1}$. Four of the pili, which have all absorbed RNA phage MS2, are terminated by vesicular knobs. The areas outlined in $(a)$ are enlarged in $(b)$ and $(c)$ where the terminal knobs are indicated by arrows. 

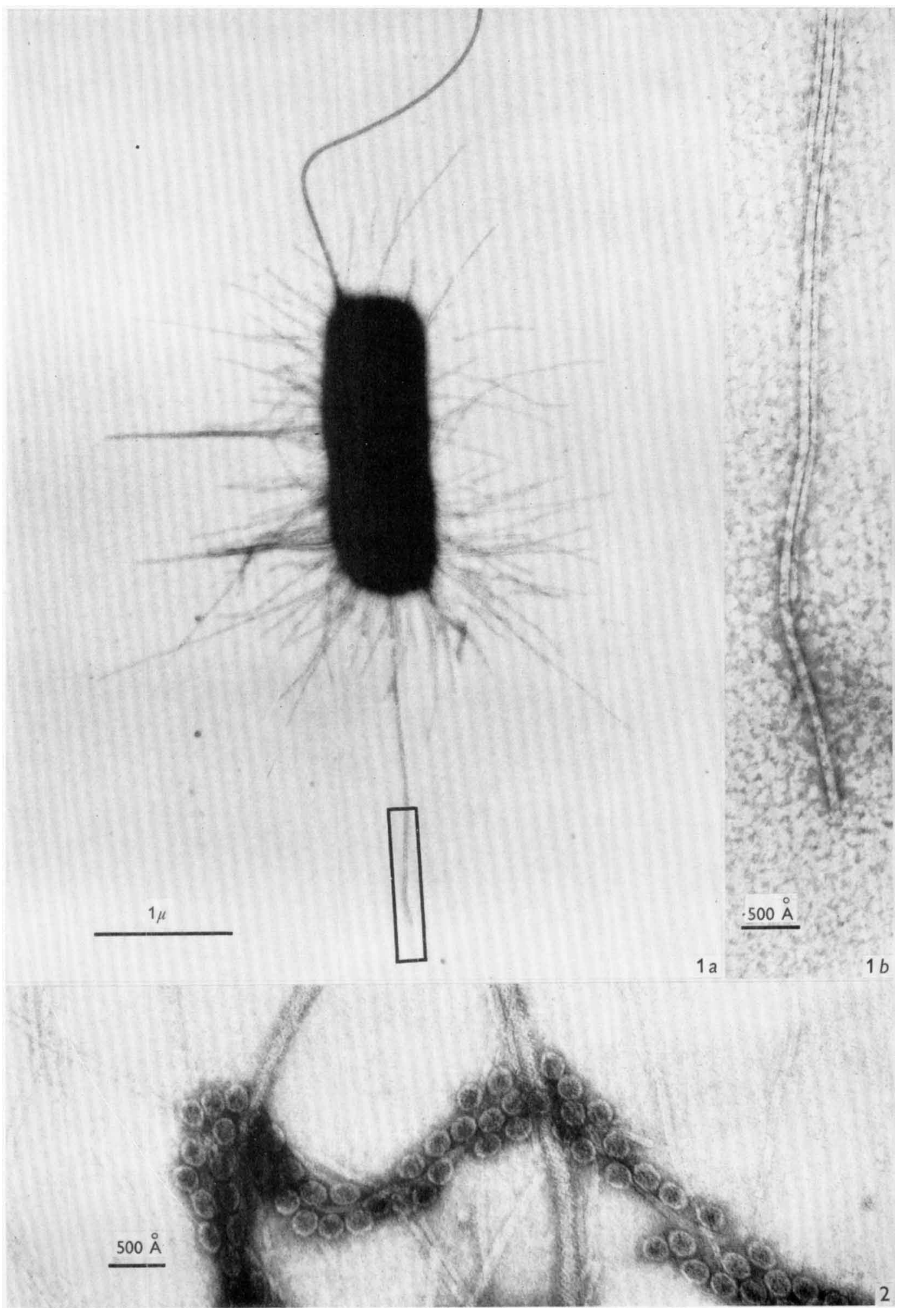
Journal of General Microbiology, Vol. 45, No. 2

Plate 2
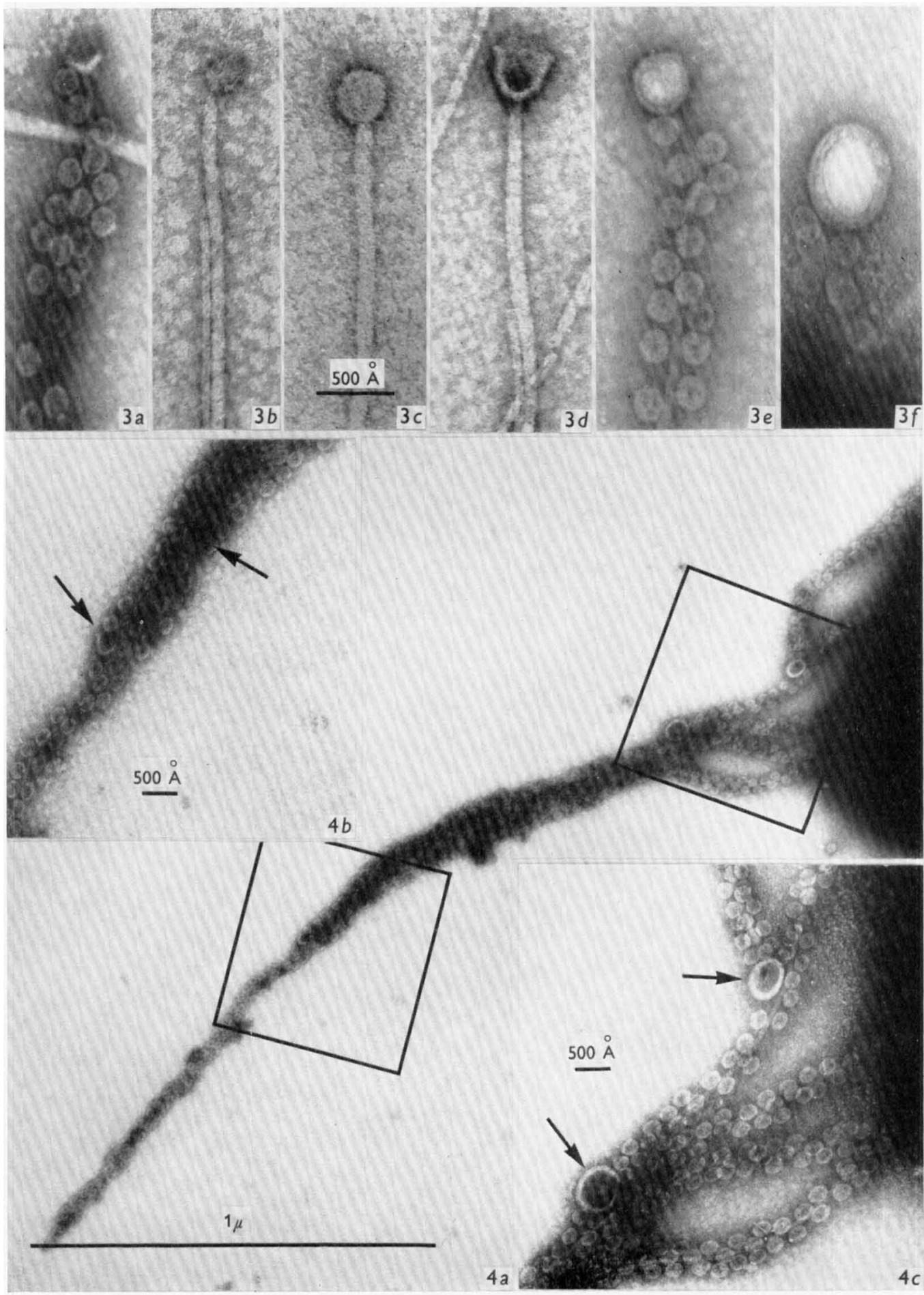

A. M. LAWN 
Journal of General Microbiology, Vol. 45, No. 2

Plate 3

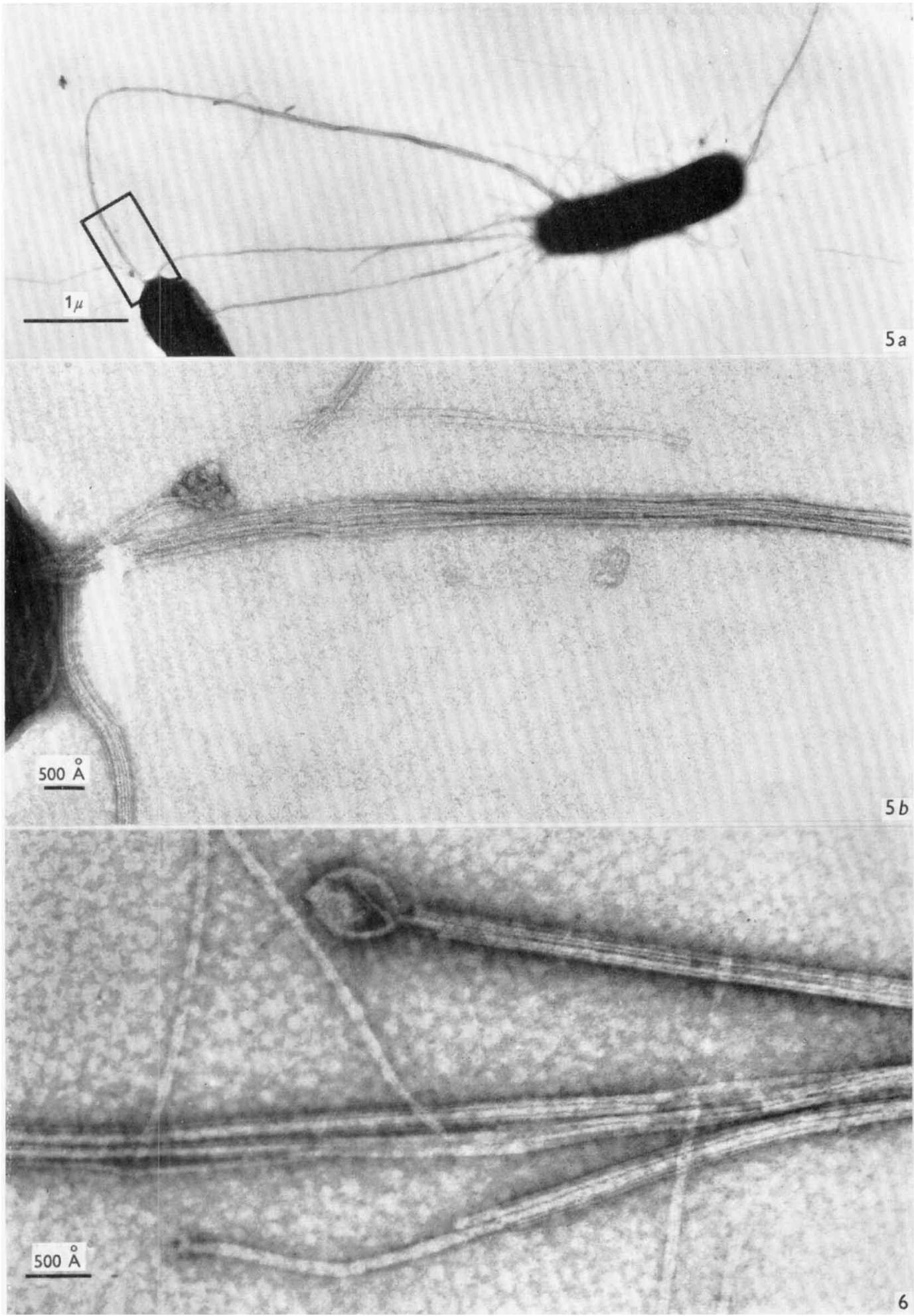

A. M. LAWN 
Journal of General Microbiology, Vol. 45, No. 2

Plate 4

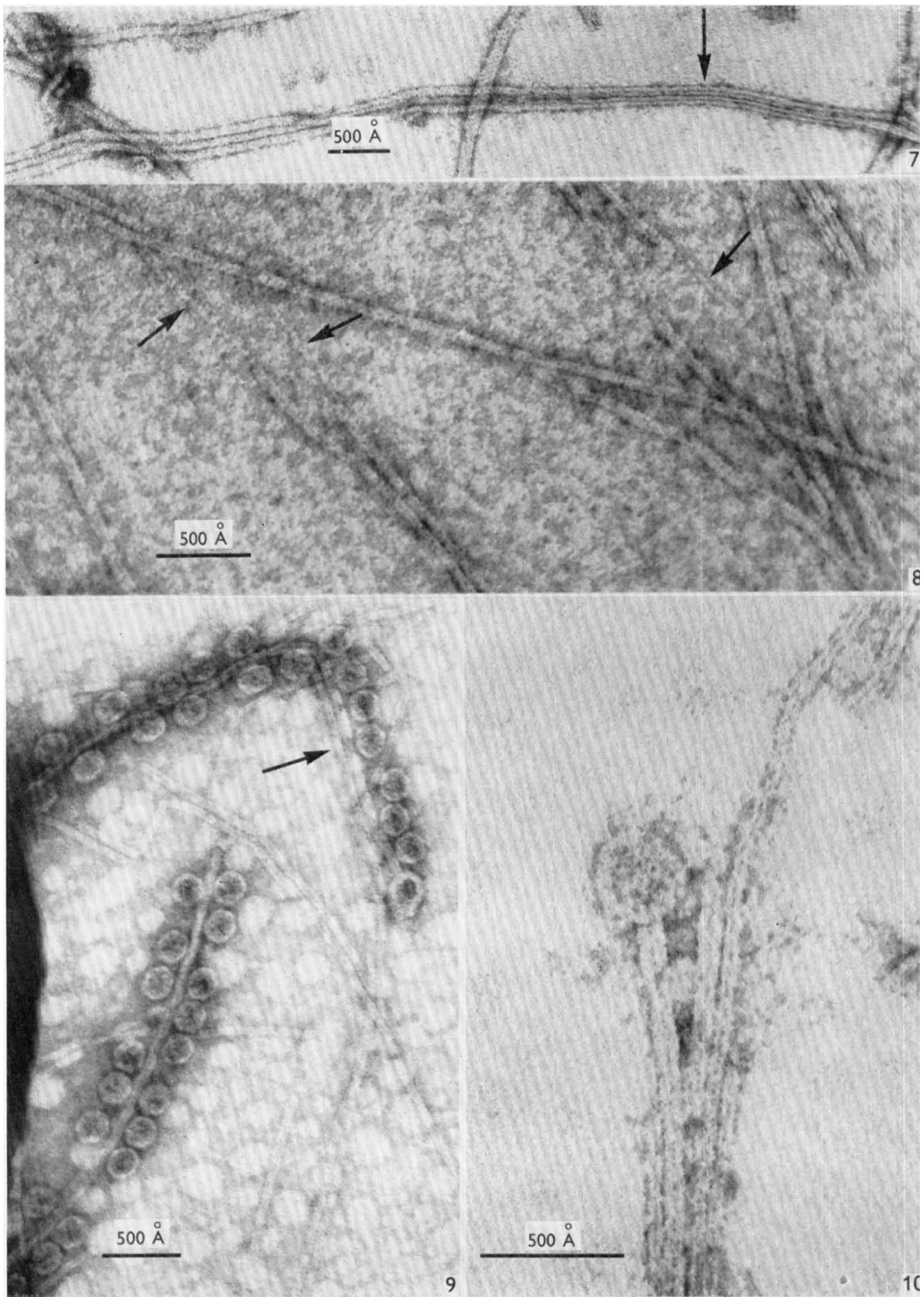

A. M. LAWN 


\section{Plate 3}

Fig. 5. The bacteria ( $E$. coli $\mathrm{K} 12 \mathrm{R} 1)$ are linked by stranded threads. Part of $(a)$ is enlarged in $(b)$ so that the strands are defined and may be compared to detached common pili in the vicinity. No phage has been added.

Fig. 6. Two F type pili from $E$. coli $\mathrm{k} 12 \mathrm{R237}$, one with a terminal knob, project from the ends of stranded bundles. The $\mathrm{F}$ type pili are wider than the common pili which ensheath them. No phage has been added.

\section{Plate 4}

Fig. 7. On the left-hand side of the figure three pili lie side by side and their central dark lines are not prominent. On the right (arrow) one of the pili is superposed on the other two, giving an illusion of two pili with unusually prominent central lines.

Fig. 8. Several of the common pili in this figure have been broken. Their ends are joined by fine filaments 15-25 $\AA$ in diameter (arrows).

Fig. 9. Where a common pilus runs along a $\mathbf{F}$ type pilus (arrow) the phage MS2 is prevented from absorbing to the latter on the associated side. In other regions $F$ type pili absorb phage equally on both sides. Note the flask-shaped terminal knob on one of the F type pili (E. coli K12 R 237).

Fig. 10. Two of these pili from $E$. coli $\mathrm{K} 12 \mathrm{R237}$ can be identified as $\mathrm{F}$ type pili because they are clearly wider than the adjacent common pili. One of the $F$ type pili has a terminal knob. The central dark line is of similar diameter in the two types of pilus. Its apparent maximum diameter is approximately $15 \AA$ (uranyl formate stain). 